\title{
Structural and electronic properties of 2D (graphene, hBN)/H-terminated diamond (100) heterostructures
}

Cite as: Appl. Phys. Lett. 117, 121901 (2020); https://doi.org/10.1063/5.0020620

Submitted: 03 July 2020 . Accepted: 11 September 2020 . Published Online: 24 September 2020

(D) Pegah S. Mirabedini, (D) Bishwajit Debnath, (D) Mahesh R. Neupane, P. Alex Greaney, A. Glen Birdwell, (D) Dmitry Ruzmetov, Kevin G. Crawford, Pankaj Shah, James Weil, Tony. G. Ivanov, et al.

\section{COLLECTIONS}

Paper published as part of the special topic on Ultrawide Bandgap Semiconductors
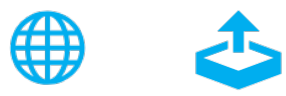

\section{ARTICLES YOU MAY BE INTERESTED IN}

High-mobility diamond field effect transistor with a monocrystalline $\mathrm{h}$-BN gate dielectric APL Materials 6, 111105 (2018); https://doi.org/10.1063/1.5055812

2D hole gas mobility at diamond/insulator interface

Applied Physics Letters 116, 162105 (2020); https://doi.org/10.1063/5.0002768

Ultrawide bandgap semiconductors

Applied Physics Letters 118, 200401 (2021); https://doi.org/10.1063/5.0055292

\section{Challenge us.} What are your needs for periodic signal detection?

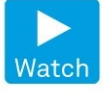

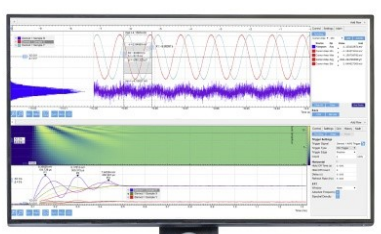

0
Zurich $\checkmark$ Instruments 


\title{
Structural and electronic properties of 2D (graphene, hBN)/H-terminated diamond (100) heterostructures
}

Cite as: Appl. Phys. Lett. 117, 121901 (2020); doi: 10.1063/5.0020620

Submitted: 3 July 2020 - Accepted: 11 September 2020 •

Published Online: 24 September 2020

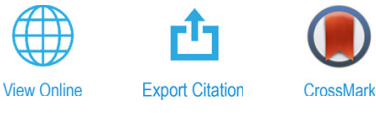

Pegah S. Mirabedini, ${ }^{1}$ (D) Bishwajit Debnath, ${ }^{2}$ (D) Mahesh R. Neupane, ${ }^{1,2,3, a)}$ (D) P. Alex Greaney, ${ }^{1,4, a)}$ A. Glen Birdwell, Dmitry Ruzmetov, ${ }^{3}$ (iD Kevin G. Crawford, ${ }^{3}$ (iD Pankaj Shah, ${ }^{3}$ James Weil, $^{3}$ and Tony. G. Ivanov ${ }^{3}$

\author{
AFFILIATIONS \\ ${ }^{7}$ Materials Science and Engineering Program, University of California, Riverside, California 92521, USA \\ ${ }^{2}$ Department of Electrical and Computer Engineering, University of California, Riverside, California 92521, USA \\ ${ }^{3}$ CCDC U.S. Army Research Laboratory, Adelphi, Maryland 21005, USA \\ ${ }^{4}$ Department of Mechanical Engineering, University of California, Riverside, California 92521, USA \\ Note: This paper is part of the Special Topic on Ultrawide Bandgap Semiconductors. \\ a) Authors to whom correspondence should be addressed: mahesh.r.neupane.civ@mail.mil and greaney@ucr.edu
}

\begin{abstract}
We report a first-principles study of the structural and electronic properties of two-dimensional (2D) layer/hydrogen-terminated diamond (100) heterostructures. Both the 2D layers exhibit weak van-der-Waals (vdW) interactions and develop rippled configurations with the $\mathrm{H}$-diamond (100) substrate to compensate for the induced strain. The adhesion energy of the hexagonal boron nitride (hBN) layer is slightly higher, and it exhibits a higher degree of rippling compared to the graphene layer. A charge transfer analysis reveals a small amount of charge transfer from the $\mathrm{H}$-diamond (100) surface to the 2D layers, and most of the transferred charge was found to be confined within the vdW gap. In the graphene/H-diamond (100) heterostructure, the semi-metallic characteristic of the graphene layer is preserved. On the other hand, the hBN/H-diamond (100) heterostructure shows semiconducting characteristics with an indirect bandgap of $3.55 \mathrm{eV}$, where the hBN layer forms a Type-II band alignment with the H-diamond (100) surface. The resultant conduction band offset and valence band offset are $0.10 \mathrm{eV}$ and $1.38 \mathrm{eV}$, respectively. A thin layer of $\mathrm{hBN}$ offers a defect-free interface with the H-diamond (100) surface and provides a layer-dependent tunability of electronic properties and band alignment for surface-doped diamond field effect transistors.
\end{abstract}

https://doi.org/10.1063/5.0020620

Diamond is an ultra-wide bandgap (UWBG) semiconductor with the highest breakdown field and carrier mobility, making it an attractive material of choice for next-generation high-speed and highpower electronic and RF device applications. ${ }^{1,2}$ However, unlike other WBGs, diamond cannot be doped with shallow dopants because of the high dopant activation energies. ${ }^{2,3}$ An advanced doping technique that has been developed to mitigate this limitation is Surface Doping (SD). ${ }^{4,5}$ In this technique, a thin film of acceptor layer with a high electron affinity (EA) is interfaced with a hydrogenated diamond (H-diamond) surface where the $\mathrm{C}-\mathrm{H}$ dipoles induce a negative electron affinity (NEA). The latter leads to an upward band bending that creates a hole accumulation region at the diamond surface. ${ }^{6}$ This accumulated charge forms a high-mobility two-dimensional hole gas (2DHG) channel, resulting in $p$-type doping of the diamond surface. The performance of SD-based devices is based on the 2DHG channel and relies on the hydrogen and acceptor layer quality. ${ }^{8}$ Initial
SD-based diamond devices used atmospheric adsorbates as acceptor layers. ${ }^{4,9}$ However, recent studies have demonstrated improved device performance utilizing relatively controllable amorphous oxide-based acceptor layers, such as $\mathrm{Al}_{2} \mathrm{O}_{3},{ }^{10} \mathrm{MoO}_{3}{ }^{11}$ and $\mathrm{V}_{2} \mathrm{O}_{5} \cdot{ }^{12,13}$ Although amorphous oxide layers provide favorable band alignment for charge transfer, they suffer from low 2DHG carrier mobilities in diamond due to interface and Coulomb scattering originated from the fixed and trapped charges at the oxide/H-diamond interface. ${ }^{14,15}$ Furthermore, high gate leakage current caused by trap-assisted tunneling ${ }^{16,17}$ limits H-diamond devices' applicability in high-field, high-power operations.

Recent studies suggest using a thin layer of $2 \mathrm{D}$ materials as the gate dielectric layer to mitigate the limitations of oxide-based acceptor layers. ${ }^{14,18}$ These studies report improvements in device parameters such as $2 \mathrm{DHG}$ channel carrier densities and mobilities. Motivated by these experiments, recent theoretical modeling based on the effective mass approach predicted that a thin layer of $2 \mathrm{D}$ layer as an interfacial 
capping layer can minimize the impact of interface roughness or even facilitate the charge transfer across the H-diamond/acceptor layer interface and improve the sheet hole concentration. ${ }^{19,20}$ Despite proof of improved device performance, theoretical effort to study the structural and electronic properties of $2 \mathrm{D} / \mathrm{H}$-diamond heterostructures with an atomistic resolution, particularly with $\mathrm{hBN}$ as the $2 \mathrm{D}$ layer, is still lacking. Furthermore, a fundamental understanding of the structural alignment and charge transfer between the 2D layer and $\mathrm{H}$-diamond is key to the successful integration of $2 \mathrm{D}$ layers in nextgeneration scalable SD-based diamond devices.

(111) and (100) facets are the most common diamond surfaces in the device community. Though the hexagonal 2D layers are crystallographically compatible with the (111) facet, the complex surface reconstruction mechanism and surface structural properties make it less desirable for the device design. In addition, this surface also suffers from high structural defects and low structural stability, ${ }^{21}$ whereas the (100) facet generally exhibits fewer defects during growth, ${ }^{22}$ and its $2 \times 1$ reconstruction is also highly stable. ${ }^{23}$ Hence, it is widely used in diamond-based RF devices. ${ }^{24-29}$ Despite these advantages, which makes (100) the most technologically important diamond surface, the lattice mismatch in the hexagonal 2D layer on the diamond complicates the computational studies. As a result, recent computational studies on 2D/diamond heterostructures have utilized the (111) diamond facet to mitigate the interfacial strain. ${ }^{30-32}$ Motivated by this, we study the structural and electronic properties resulting from the integration of hBN and graphene on the H-diamond (100) surface using the first principles simulations. Details on the computed structural models and the adapted methods are described in the supplementary material (SI).

Figures 1(a) and 1(b) depict top-views of the graphene/ $\mathrm{H}$-diamond (100) [hereafter, called $\mathrm{G} / \mathrm{D}(100)]$ and $\mathrm{hBN} / \mathrm{H}$-diamond (100) [hereafter, called hBN/D(100)] heterostructures, respectively. After undergoing the transformation to bring the $2 \mathrm{D}$ layer into registry with the H-diamond (100) surface, the heterostructures, with an optimized vdW-gap $(\delta)$ of $\sim 3 \AA$, were fully relaxed during which both the $\mathrm{hBN}$ and graphene sheets developed a corrugation perpendicular to the compressive axis, as shown in Figs. 1(c) and 1(d). The corrugated (buckled) alignment observed here occurs to relax residual compressive strain imposed by the periodicity of the supercell. Similar substrate-induced corrugation or rippling of the graphene layer was also observed in the epitaxial growth of graphene on $\mathrm{Ge}^{33}$ and $\mathrm{SiC}$ substrates, ${ }^{34}$ and a DFT study of graphene on the diamond (111) surface is carried out. ${ }^{35}$ Though this atomic distortion results in the modification of the local potential within the layer, it has a minimal effect on the electronic properties as discussed in the supplementary material.

To correlate the magnitude of rippling of the $2 \mathrm{D}$ layers with the layer adhesion energy, the adhesion energy per atom $\left(E_{A}\right)$ is evaluated using the equation $E_{A}=\frac{1}{n}\left(E_{S / D}-E_{S}-E_{D}\right),{ }^{35}$ where $E_{D}, E_{S}$, and $E_{S / D}$ are the total energy of $\mathrm{H}$-diamond (100), the 2D layer, and the 2D/H-diamond (100) heterostructure, respectively. The term $n$ is the number of atoms in the 2D layer. The per atom adhesion energy of graphene on the H-diamond (100) surface was found to be $-0.0314 \mathrm{eV}$, which is comparable to the reported value $(-0.05 \mathrm{eV})$ for the similar system. ${ }^{30}$ The variation in the adsorption energy values comes from the difference in the interfacial strain due to the unreconstructed and bulk-like used in Ref. 30, which is distinctly different from the experimentally observed, reconstructed $(2 \times 1)$ surface used (a)

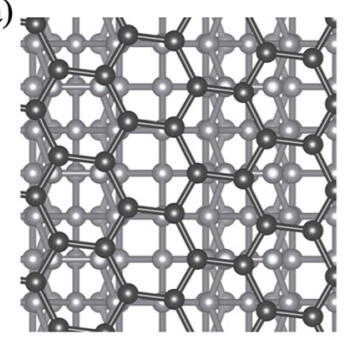

(c)

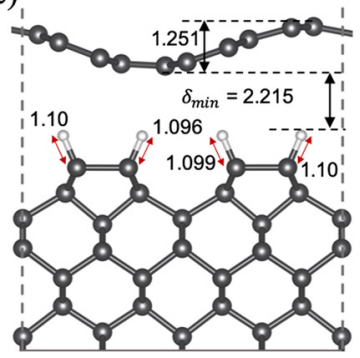

(b)

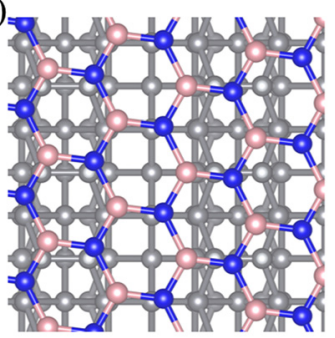

(d)

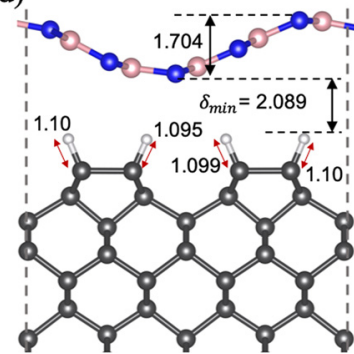

FIG. 1. Atomistic structures of 2D/H-diamond (100) heterostructures. Top and side views of the optimized (a) and (c) graphene/H-diamond (100), i.e., $G / D(100)$, and (b) and (d) hBN/H-diamond (100), i.e., hBN/D(100), heterostructures. The C-H bond lengths, peak to valley distance of the rippling curvature, and the minimum $\mathrm{vdW}$ spacing after rippling $\left(\boldsymbol{\delta}_{\min }\right)$ values are annotated in the figures. All distances are in $\AA$. The hydrogen, carbon, boron, and nitrogen atoms are shown with white, gray, pink, and blue colors, respectively.

in this study. For hBN on the H-diamond (100) system, the observed per atom adhesion energy was $-0.0396 \mathrm{eV}$, indicating a slightly stronger interaction between the hBN layer and the $\mathrm{H}$-diamond surface. In both cases, the trough of the ripple was aligned between the rows of reconstructed $\mathrm{C}-\mathrm{C}$ bonds on the substrate. The minimum vdW-gaps $\left(\delta_{\text {min }}\right)$ at the valley of the corrugation are $2.215 \AA$ and $2.089 \AA$ for graphene and hBN, respectively, as illustrated in Figs. 1(c) and 1(d). The amplitude of the observed ripple in graphene is smaller than that of $\mathrm{hBN}$ due to the lower compressive strain imposed on the graphene layer required to make it commensurate with the diamond surface.

The bending of the $\mathrm{sp}^{2}$ bonds in the rippled sheets distorts the orbital hybridization of the atoms in the locations where the curvature is large, leaving orbitals free to participate in bonding with the substrate. This effect is larger in hBN than in graphene as the higher compressive strain in $\mathrm{hBN}$ imposes a tighter radius of curvature in this sheet's ripples. Therefore, the interaction between the hBN layer and the substrate is stronger.

In order to correlate the observed adhesion strength with the degree of charge transfer between the 2D layer and the $\mathrm{H}$-diamond surface, we have evaluated the interface charge between the 2D layers and $\mathrm{H}$-diamond (100) by calculating the charge density difference using the equation $\Delta \rho=\rho_{S / D}-\rho_{D}-\rho_{S}$, where $\rho_{S / D}, \rho_{D}$, and $\rho_{S}$ represent the electron density of the heterostructure, diamond, and $2 \mathrm{D}$ layer, respectively. The $\Delta \rho$ values for the $\mathrm{G} / \mathrm{D}(100)$ and $\mathrm{hBN} / \mathrm{D}(100)$ heterostructures are shown in Figs. 2(a) and 2(b), respectively. Here, the areas enclosed by yellow (blue) isosurfaces represent the regions with the charge increase (decrease) due to interaction. Regardless of the type of $2 \mathrm{D}$ layer, the bulk of the charge transfer occurs around the valley of the ripple. A higher local charge transfer from the 
(a)

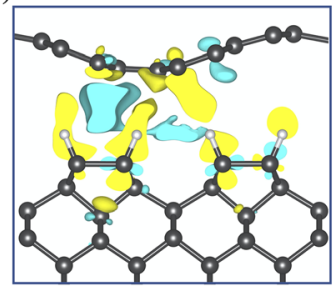

(b)

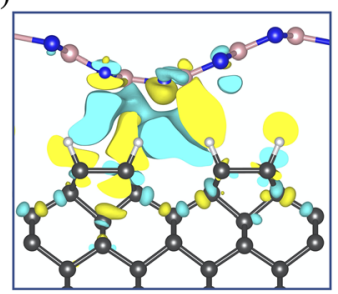

FIG. 2. Side view of the three dimensional charge density difference maps for (a) $\mathrm{G} / \mathrm{D}(100)$ and $(\mathrm{b}) \mathrm{hBN} / \mathrm{D}(100)$ heterostructures. Blue and yellow regions represent the charge loss and gain in the space with respect to the isolated 2D layer and $\mathrm{H}$ diamond (100), respectively. An isosurface value of 0.001 was used.

$\mathrm{H}$-diamond (100) to $\mathrm{hBN}$ in hBN/D (100) is observed, resulting in stronger adhesion between the layer and the H-diamond surface. The higher charge transfer and adhesion energy for the hBN/D (100) system can be attributed to the larger degree of rippling in the hBN layer.

Using the optimized geometries and corresponding self-consistent charges, we calculated the band structures of 2D/ H-diamond (100) heterostructures (Fig. 3). The band structure of the pristine rippled graphene supercell is overlaid over that of $\mathrm{G} / \mathrm{D}(100)$ in order to analyze the effect of heterogeneity on the shape and slope of the Dirac cone at $\mathrm{K}^{\prime}$, as illustrated in Fig. 3(a). The overall characteristic of the singlelayer graphene is preserved in $\mathrm{G} / \mathrm{D}(100)$, while the primary Dirac point is shifted below the Fermi level by $0.13 \mathrm{eV}$. This generates weak n-type doping of the graphene layer, indicating the effect of charge transfer across the vdW-interface [Fig. 3(a) inset]. The magnitude of graphene doping is consistent with the recent experimental report on the graphene/H-terminated (111) system. ${ }^{36,37}$ This charge transfer-induced doping of pristine graphene is consistent with earlier studies on graphene-based vdW heterostructures. ${ }^{38-42}$ Even though an intrinsic, defect-free graphene layer is gapless, a small gap opening of $10 \mathrm{meV}$ was observed around the Dirac cone for both the rippled graphene supercell and $\mathrm{G} / \mathrm{D}(100)$. The small gap around the $\mathrm{K}^{\prime}$ point, which is less than half of the room-temperature thermal energy, is consistent with other works where the induced gap is attributed to the hybridization of $\pi$ and $\sigma$ orbitals $^{30,43,44}$ and the spatially varying potentials ${ }^{45,46}$ introduced by strain and ripples. If the graphene layer is grown large enough or the substrate is designed to minimize the strain, this gap can
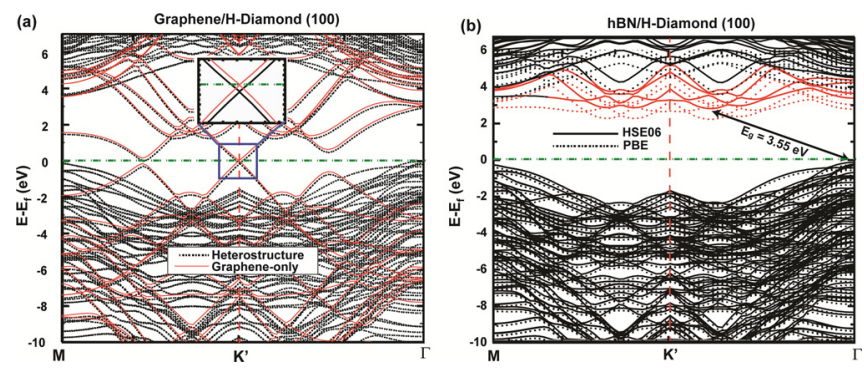

FIG. 3. Band structures for (a) G/D(100) and (b) hBN/D(100) heterostructures. In (a), the red solid and black dotted lines represent the band structures for the rippled graphene supercell and $G / D(100)$ heterostructure, respectively. In (b), the solid and dotted lines represent the band structures for $h B N / D(100)$ using HSE06 and PBE functionals. Contributions from the $\mathrm{hBN}$ layer to the $\mathrm{CBE}$ states are presented with the light (red) lines. be suppressed, and hence, the $\mathrm{G} / \mathrm{D}(100)$ heterostructure can be deemed as a semi-metallic system. This observation is consistent with the previous studies on graphene-based heterostructures..$^{30,34,47}$

The band structure for $\mathrm{hBN} / \mathrm{D}(100)$ is illustrated in Fig. 3(b). Here, to highlight the role of the exchange-correlation term in the band edges, we plotted the band structures calculated from both the local functional (PBE) and the hybrid functional (HSE06). The HSE06 functional increases the fundamental energy gap $\left(\mathrm{E}_{\mathrm{g}}\right)$ of $\mathrm{hBN} / \mathrm{D}(100)$ by $\sim 30 \%$ to $3.5 \mathrm{eV}$ compared to the PBE functional. The main contributions to the scaling originate from the $\mathrm{CB}$ states. Similar exchangecorrelation related up-scaling of energy gaps was observed for the bulk diamond and H-diamond (100) systems. Quantitatively, the energy gaps increased from 4.2 and $2.6 \mathrm{eV}$ (PBE) to $5.3 \mathrm{eV}$ and $4.2 \mathrm{eV}$ (HSE06) for bulk diamond and H-diamond (100), respectively. Other theoretical studies on wide bandgap semiconductors also reported similar degrees of scaling for the fundamental gaps. ${ }^{48,49}$ Though $\mathrm{H}$-diamond (100) has a direct bandgap with CB minima (CBM) and VB maxima (VBM) located at the $\Gamma$-point, a direct-to-indirect energy gap transition occurs in the $\mathrm{hBN} / \mathrm{D}(100)$ system due to the heterogeneity so that the CBE is located between the $\mathrm{K}^{\prime}$ and $\Gamma$-points and the $\mathrm{VBE}$ is placed at the $\Gamma$-point of the BZ.

One can gain further insight into the role of the proximal 2D layers in modifying device-related properties by analyzing the site projected partial density of states (PDOS) of the 2D/H-diamond (100) heterostructures. In G/D(100), Fig. 4(a), most of the contributions to the two peaks around the Fermi level are from the graphene layer, which is consistent with the observed Dirac cones around the Fermi level seen in the band structure plot in Fig. 3(a). The VB states at the $\Gamma$-point of this system are co-located in the vicinity of the $\mathrm{C}-\mathrm{H}$ bonds on the diamond surface and the graphene layer, as illustrated in Fig. S1(a) in the supplementary material. These results suggest that graphene could be a very promising material as the metal electrode, and it can enhance the contact performance while maintaining the intrinsic electronic properties of the interfaced diamond surface.

In contrast to the $\mathrm{G} / \mathrm{D}(100)$ system, the $\mathrm{hBN} / \mathrm{D}(100)$ system exhibits semiconducting properties with a significant energy gap of $3.55 \mathrm{eV}$, as shown in Fig. 3(b). Although the energy gap is $\sim 15 \%$ smaller than the gap of isolated H-diamond (100), it is comparable to other WBG semiconductors such as $\mathrm{GaN}(3.4 \mathrm{eV})$ and $\mathrm{SiC}(3.3 \mathrm{eV}) .^{50}$ Here, the hybridized states from the surface and bulk $\mathrm{C}$ atoms mainly contribute to the VBE states. On the other hand, the $\boldsymbol{p}_{\boldsymbol{z}}$ orbitals from the $\mathrm{B}$ atoms in the hBN layer contribute to the CBE states and are consequently localized around $\mathrm{B}$ atoms in the $\mathrm{hBN}$ layer. This is further elucidated by the band decomposed charge densities around the band edges, illustrated in Figs. S1(c) and S1(d) in the supplementary material. This spatial separation of electrons and holes by more than $5 \AA$ in $\mathrm{hBN} / \mathrm{D}(100)$ is highly desirable for minimizing the Coulomb scattering in the channel of SD-based diamond transistors. ${ }^{18}$

In the semi-metallic nature of the $\mathrm{G} / \mathrm{D}(100)$ system, there are no gap states originating from the graphene layer in the $\mathrm{H}$-diamond (100) gap. As a result, the Fermi level pinning is minimal, and the graphene layer is expected to act as a metal contact. ${ }^{51,52}$ Here, the n-type (p-type) Schottky barrier, ${ }^{53,54} \Phi_{n}\left(\Phi_{p}\right)$, is defined as the energy difference between the CBE (VBE) state of H-diamond (100) and the Dirac point at the $\mathrm{K}^{\prime}$-point. The observed $\Phi_{p}$ and $\Phi_{n}$ values are $13 \mathrm{meV}$ and $1.73 \mathrm{eV}$, respectively. The relatively small $\Phi_{p}$ value indicates that the graphene layer in $\mathrm{G} / \mathrm{D}(100)$ acts as a p-type Ohmic contact. Since the 

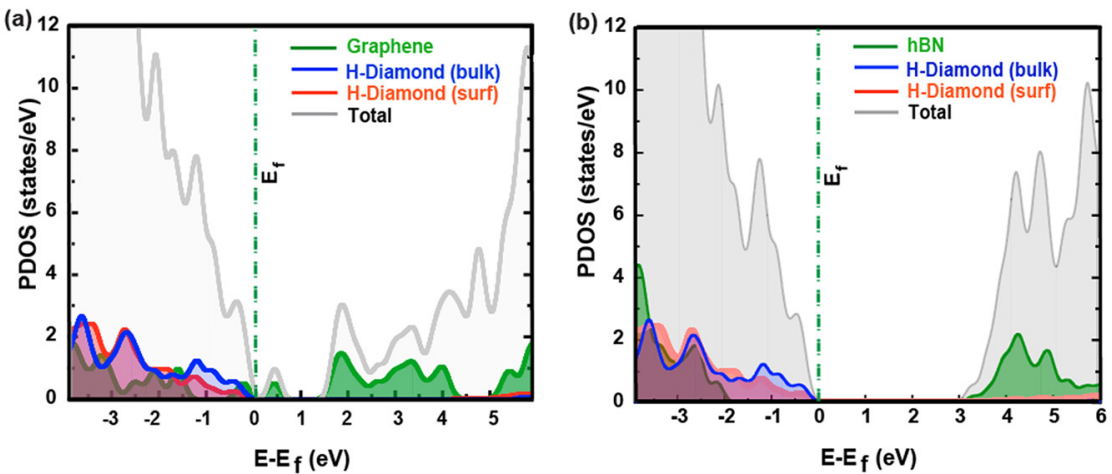

(c)

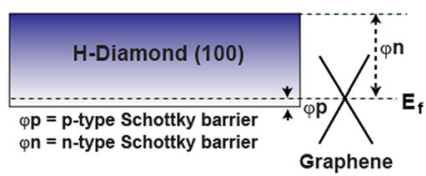

TYPE-II band Alignment

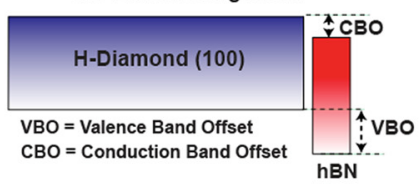

FIG. 4. Partial density of state (PDOS) plots of the surface and bulk atoms on the diamond (100) surface and the constituent layer for (a) G/D(100) and (b) hBN/D(100) systems; (c) band alignment schematics for both heterostructures.

$\mathrm{H}$-diamond (100) band edges are sensitive to the surface and termination types, $\mathrm{G} / \mathrm{D}(100)$ exhibits tunable Schottky and Ohmic contacts with promising device applications based on unique and complementary intrinsic properties of graphene and $\mathrm{H}$-diamond (100).

In the $\mathrm{hBN} / \mathrm{D}(100)$ system, the CBE and VBE energy levels are primarily composed of the states from the hBN and H-diamond (100) systems, respectively. Furthermore, their energy levels form a Type-II (staggered) band alignment, as illustrated in the bottom panel of Fig. 4(c). This type of band alignment allows the confinement of electrons and holes in different spatial locations of the heterojunction and is widely used in memory ${ }^{55}$ and unipolar electronic device ${ }^{56}$ applications. The observed $\mathrm{CBO}$ and $\mathrm{VBO}$ values are $0.1 \mathrm{eV}$ and $1.38 \mathrm{eV}$, respectively. This indicates higher confinement for holes (vis-à-vis electrons), in the $\mathrm{hBN} / \mathrm{D}(100)$ system. Quantitatively, the observed $\mathrm{VBO}$ value is lower than the reported value for the $\mathrm{Al}_{2} \mathrm{O}_{3} / \mathrm{H}$-diamond (100) system obtained by analyzing the energy difference between the site-resolved core-level shifts in XPS measurements. ${ }^{10}$ Despite this, an $\mathrm{hBN}$ layer interfaced with $\mathrm{H}$-diamond offers the advantage of having a low number of charge impurities and dangling bonds. Besides, an hBN layer between the $\mathrm{H}$-diamond and the acceptor layer may serve to protect the hydrogenated diamond surface and thereby improves the stability of the hole channel preventing charge leakage during the device operation. The hBN/D(100) heterostructure is also an interesting system to realize interlayer indirect excitons because of the small screening effect and heavy carrier masses in the constituent hBN and H-diamond systems. Furthermore, because of the Type-II band alignment between the $\mathrm{hBN}$ and H-diamond (100) systems, the spatially separated electrons and holes, as illustrated in Figs S1(c) and S1(d), form indirect excitons that are strongly bound and are long-lived, as compared to the excitons realized in III-V and 2D heterostructures. ${ }^{57-59}$

In this study, we performed systematic DFT calculations and analyzed the structural and electronic properties of $2 \mathrm{D} / \mathrm{H}$-diamond (100) heterostructures. Both the hBN and graphene layers are physisorbed on the H-diamond (100) surface through vdW-like interactions and exhibit a structural corrugation around the reconstructed diamond surface. The corrugation is an artifact of the in-plane strain between the 2D layers and the H-diamond (100) surface and was found to have little or no effect on the electronic properties. Though the charge transfer from the H-diamond (100) to either of the 2D layers is minimal, the charge transfer occurs through the valley of rippling, and the majority of transferred charges are confined within the vdW gaps between the 2D layers and the H-diamond (100) surface. The $\mathrm{G} / \mathrm{D}(100)$ system retains the linear band dispersion characteristic of the graphene layer and exhibits semi-metallic properties. Conversely, the $\mathrm{hBN} / \mathrm{D}(100)$ heterostructure maintains the wide bandgap characteristics of each constituent system with an energy gap of $3.35 \mathrm{eV}$. Band alignment analysis reveals a Type-II band alignment between the $\mathrm{hBN}$ layer and the H-diamond (100) surface with the $\mathrm{CBO}$ and $\mathrm{VBO}$ of $0.10 \mathrm{eV}$ and $1.38 \mathrm{eV}$, respectively. A significant finding of this work is that a thin layer of hBN not only offers a defect-free interface with the H-diamond (100) surface but also provides a layerdependent tunability of electronic properties and band alignment with the acceptor layer in SD-based diamond devices.

See the supplementary material for the details on methods and models used, discussion on the effect of 2D layer rippling on electronic properties, and band-decomposed partial charge density plots.

\section{DATA AVAILABILITY}

The data that support the findings of this study are available from the corresponding author upon reasonable request.

\section{REFERENCES}

'J. Isberg, J. Hammersberg, E. Johansson, T. Wikström, D. J. Twitchen, A. J. Whitehead, S. E. Coe, and G. A. Scarsbrook, Science 297, 1670 (2002).

${ }^{2}$ M. Kasu, K. Ueda, H. Kageshima, and Y. Yamauchi, Diamond Relat. Mater. 17, 741 (2008).

${ }^{3}$ E. Kohn and A. Denisenko, Thin Solid Films 515, 4333 (2007).

${ }^{4}$ F. Maier, M. Riedel, B. Mantel, J. Ristein, and L. Ley, Phys. Rev. Lett. 85, 3472 (2000).

${ }^{5}$ P. Strobel, M. Riedel, J. Ristein, and L. Ley, Nature 430, 439 (2004).

${ }^{6}$ M. T. Edmonds, C. I. Pakes, S. Mammadov, W. Zhang, A. Tadich, J. Ristein, and L. Ley, Appl. Phys. Lett. 98, 102101 (2011).

${ }^{7}$ J. Ristein, Science 313, 1057 (2006).

${ }^{8}$ Z. Yin, M. Tordjman, Y. Lee, A. Vardi, R. Kalish, and J. A. Del Alamo, Sci. Adv. 4, eaau0480 (2018).

${ }^{9}$ M. Kasu, M. Kubovic, A. Aleksov, N. Teofilov, Y. Taniyasu, R. Sauer, E. Kohn, T. Makimoto, and N. Kobayashi, Diamond Relat. Mater. 13, 226 (2004).

${ }^{10}$ Y. Yang, F. A. Koeck, M. Dutta, X. Wang, S. Chowdhury, and R. J. Nemanich, J. Appl. Phys. 122, 155304 (2017).

${ }^{11}$ S. A. O. Russell, L. Cao, D. Qi, A. Tallaire, K. G. Crawford, A. T. S. Wee, and D. A. J. Moran, Appl. Phys. Lett. 103, 202112 (2013). 
${ }^{12}$ K. G. Crawford, L. Cao, D. Qi, A. Tallaire, E. Limiti, C. Verona, A. T. S. Wee, and D. A. J. Moran, Appl. Phys. Lett. 108, 042103 (2016).

${ }^{13}$ K. G. Crawford, J. D. Weil, P. B. Shah, D. A. Ruzmetov, M. R. Neupane, K. Kingkeo, A. G. Birdwell, and T. G. Ivanov, IEEE Trans. Electron Devices 67, 2270 (2020).

${ }^{14}$ Y. Sasama, K. Komatsu, S. Moriyama, M. Imura, T. Teraji, K. Watanabe, T. Taniguchi, T. Uchihashi, and Y. Takahide, APL Mater. 6, 111105 (2018).

${ }^{15}$ H. Kawarada, T. Yamada, D. Xu, H. Tsuboi, Y. Kitabayashi, D. Matsumura, M. Shibata, T. Kudo, M. Inaba, and A. Hiraiwa, Sci. Rep. 7, 42368 (2017).

${ }^{16}$ L. Colalongo, M. Valdinoci, G. Baccarani, P. Migliorato, G. Tallarida, and C. Reita, Solid-State Electron. 41, 627 (1997).

${ }^{17}$ A. Alnuaimi, K. Islam, and A. Nayfeh, Sol. Energy 98, 236 (2013).

${ }^{18}$ Y. Sasama, K. Komatsu, S. Moriyama, M. Imura, S. Sugiura, T. Terashima, S. Uji, K. Watanabe, T. Taniguchi, T. Uchihashi, and Y. Takahide, Phys. Rev. Mater. 3, 121601 (2019).

${ }^{19}$ Z. Ren, D. Lv, J. Xu, J. Zhang, J. Zhang, K. Su, C. Zhang, and Y. Hao, Appl. Phys. Lett. 116, 013503 (2020).

${ }^{20}$ G. Daligou and J. Pernot, Appl. Phys. Lett. 116, 162105 (2020).

${ }^{21}$ C. Wild, R. Kohl, N. Herres, W. Muller-Sebert, and P. Koidl, Diamond Relat. Mater. 3, 373 (1994).

${ }^{22}$ G. Bogdan, M. Nesladek, J. D’Haen, K. Haenen, and M. D'Olieslaeger, Diamond Relat. Mater. 15, 508 (2006).

${ }^{23}$ T. Frauenheim, U. Stephan, P. Blaudeck, and D. Porezag, Phys. Rev. B 48, 18189 (1993).

${ }^{24}$ Y. M. Wang, K. W. Wong, S. T. Lee, M. Nishitani-Gamo, I. Sakaguchi, K. P. Loh, and T. Ando, Diamond Relat. Mater. 9, 1582 (2000).

${ }^{25}$ M. Kasu, K. Ueda, H. Ye, Y. Yamauchi, and S. Sasaki, Electron. Lett. 41, 1249 (2005).

${ }^{26}$ K. Hirama, S. Miyamoto, H. Matsudaira, K. Yamada, and H. Kawaradaa, Appl. Phys. Lett. 88, 112117 (2006).

${ }^{27}$ M. Kasu, K. Ueda, Y. Yamauchi, A. Tallaire, and T. Makimoto, Diamond Relat. Mater. 16, 1010 (2007).

${ }^{28}$ P. B. Shah, J. Weil, A. G. Birdwell, and T. Ivanov, MRS Advances 2(41), 2235-2240 (2017).

${ }^{29}$ M. Attrash, M. K. Kuntumalla, and A. Hoffman, Surf. Sci. 681, 95 (2019).

${ }^{30}$ W. Hu, Z. Li, and J. Yang, J. Chem. Phys. 138, 054701 (2013).

${ }^{31}$ S. Zhao and K. Larsson, Diamond Relat. Mater. 66, 52 (2016).

${ }^{32}$ Y. Ma, Y. Dai, M. Guo, and B. Huang, Phys. Rev. B 85, 235448 (2012).

${ }^{33}$ C. D. Mendoza, N. S. Figueroa, M. E. H. Maia da Costa, and F. L. Freire, Jr., Sci. Rep. 9, 12547 (2019).

${ }^{34}$ F. Varchon, P. Mallet, J. Y. Veuillen, and L. Magaud, Phys. Rev. B 77, 235412 (2008).

${ }^{35}$ D. Selli, I. Baburin, S. Leoni, Z. Zhu, D. Tománek, and G. Seifert, J. Phys.: Condens. Matter 25, 435302 (2013).

${ }^{36}$ G. Wan, S. Panditharatne, N. A. Fox, and M. Cattelan, Nano Express 1, 020011 (2020).
${ }^{37}$ T. Yamada, T. Masuzawa, H. Mimura, and K. Okano, Appl. Phys. Lett. 114, 231601 (2019).

${ }^{38}$ W. Hu, Z. Li, and J. Yang, J. Chem. Phys. 139, 154704 (2013).

${ }^{39}$ H. Tan, Y. Fan, Y. Rong, B. Porter, C. S. Lau, Y. Zhou, Z. He, S. Wang, H. Bhaskaran, and J. H. Warner, ACS Appl. Mater. Interfaces 8, 1644 (2016).

${ }^{40}$ F. Lin, S. W. Chen, J. Meng, G. Tse, X. W. Fu, F. J. Xu, B. Shen, Z. M. Liao, and D. P. Yu, Appl. Phys. Lett. 105, 073103 (2014).

${ }^{41}$ R. H. Miwa, T. M. Schmidt, W. L. Scopel, and A. Fazzio, Appl. Phys. Lett. 99, 163108 (2011).

${ }^{42}$ S. Kopylov, A. Tzalenchuk, S. Kubatkin, and V. I. Fal'Ko, Appl. Phys. Lett. 97, 112109 (2010).

${ }^{43}$ U. Monteverde, J. Pal, M. A. Migliorato, M. Missous, U. Bangert, R. Zan, R. Kashtiban, and D. Powell, Carbon 91, 266 (2015).

${ }^{44}$ J. Zhang, K. P. Ong, and P. Wu, J. Phys. Chem. C 114, 12749 (2010).

${ }^{45}$ C. Park, L. Yang, Y. Son, M. L. Cohen, and S. G. Louie, Nat. Phys, 4, 213 (2008).

${ }^{46}$ W. Bao, F. Miao, Z. Chen, H. Zhang, W. Jang, C. Dames, and C. N. Lau, Nat. Nanotechnol, 4, 562 (2009).

${ }^{47}$ L. Huang, Q. Yue, J. Kang, Y. Li, and J. Li, J. Phys.: Condens. Matter 26, 295304 (2014).

${ }^{48}$ M. A. L. Marques, J. Vidal, M. J. T. Oliveira, L. Reining, and S. Botti, Phys. Rev. B 83, 035119 (2011).

${ }^{49}$ W. Chen and A. Pasquarello, Phys. Rev. B 86, 035134 (2012).

${ }^{50}$ J. Y. Tsao, S. Chowdhury, M. A. Hollis, D. Jena, N. M. Johnson, K. A. Jones, R. J. Kaplar, S. Rajan, C. G. Van de Walle, E. Bellotti, C. L. Chua, R. Collazo, M. E. Coltrin, J. A. Cooper, K. R. Evans, S. Graham, T. A. Grotjohn, E. R. Heller, M. Higashiwaki, M. S. Islam, P. W. Juodawlkis, M. A. Khan, A. D. Koehler, J. H. Leach, U. K. Mishra, R. J. Nemanich, R. C. N. Pilawa-Podgurski, J. B. Shealy, Z. Sitar, M. J. Tadjer, A. F. Witulski, M. Wraback, and J. A. Simmons, Adv. Electron. Mater. 4, 1600501 (2018).

${ }^{51}$ M. Sun, J. P. Chou, Q. Ren, Y. Zhao, J. Yu, and W. Tang, Appl. Phys. Lett. 110, 173105 (2017).

${ }^{52}$ D. Tomer, S. Rajput, L. J. Hudy, C. H. Li, and L. Li, Appl. Phys. Lett. 105, 021607 (2014).

${ }^{53}$ W. Schottky, Z. Phys. 113, 367 (1939).

${ }^{54}$ N. F. Mott, Proc. R. Soc. London, Ser. A 171, 27 (1939).

${ }^{55}$ M. R. Neupane, R. K. Lake, and R. Rahman, J. Appl. Phys. 112, 024326 (2012).

${ }^{56}$ M. Sun, J. P. Chou, J. Yu, and W. Tang, Phys. Chem. Chem. Phys. 19, 17324 (2017).

${ }^{57}$ T. Olsen, S. Latini, F. Rasmussen, and K. S. Thygesen, Phys. Rev. Lett. 116, 056401 (2016).

${ }^{58}$ E. V. Calman, M. M. Fogler, L. V. Butov, S. Hu, A. Mishchenko, and A. K. Geim, Nat. Commun. 9, 1895 (2018).

${ }^{59}$ G. Wang, A. Chernikov, M. M. Glazov, T. F. Heinz, X. Marie, T. Amand, and B. Urbaszek, Rev. Mod. Phys. 90, 21001 (2018). 